\title{
A conceptual model of subjectively rational choice
}

\author{
Gennady P. Vinogradov \\ Tver state technical University \\ Tver, Russia \\ wgp272ng@mail.ru
}

\begin{abstract}
The article considers the problem of constructing a model of decision making taking into account the individual contribution of people in a decision situation. Based on the concept of goal-oriented situation it introduces a linguistic evaluation of its components. This approach allows integrating psychological and mathematical models.
\end{abstract}

Keywords-modeling, the decision situation, decision making model, agent, multi-agent system

\section{INTRODUCTION}

Human behavior (hereinafter goal-oriented agent - GA) is the result of man's cognitive activity. In psychology cognitive activity is regarded as a process of forming a mental image (the result of conception) and a model of cognizable and analyzed objects, processes, phenomena. According to a model formed and based on the mental sets, rules, customs, past experience and knowledge a person ideates his attitude to the observable and analyzable. Then, depending on this attitude and the assessment of the object adequacy to the result of mental image and analysis, a person constructs his behavior and develops the program of his actions beginning from participation (or fractional participation) to a complete non-participation in the events, processes, phenomena which he considers important. Therefore, one possible approach to overcome shortcomings of traditional methods of describing the agent behavior in organizational systems is a rational behavior model modification. That is achieved by implicating of so-called unobservables which are human behavior characteristics used in psychology, sociology, etc. For example we introduce parameter $r$ which characterizes the type of agent, with $r \in B$ ( $r$ might be termed an agent characteristic) [1]. The objective agent function $f$ depends on the ambient environment $\theta \in \Omega$, performance $o \in A$ based on the agent mode of action $c \in C$, centrogenous control $u \in U$ and the type of agent $r$. Following the hypothesis of agent' rational behavior, the agent tries to get a result $o^{*} \in A_{O}$ which maximizes his objective function

$$
o^{*}=\operatorname{Arg} \max f(o, u, c, \theta, r) ; o \in A, c \in C \text {. }
$$

This permits to speculate about function $f$ regarding $s=F\left(\theta \in \Omega_{O}, z=G(o) \in A_{O}, q=H(c) \in C\right)$. Such an approach is to use some idealized construct (model) that describes the behavior of GA in decision-making.

\section{GOAL-ORIENTED MODEL}

Goal-oriented behavior is always associated with the decision which is made in the goal-oriented condition [2-4]. Goal-oriented condition consists of the following components:
- Behaver making a decision (GA), $k \in K$;

- Decision environment $(S)$, which refers to the set of elements and their intrinsic properties, (changes in any of them can cause or produce a change in the condition of goal-oriented decision;

- Available modes of action $C_{i j}^{k}, j=\overline{1, n}$ of the kth agent, which are at his disposal and can be used to achieve the $i$-th result (also called alternatives);

- Possible results under environment $(S)$ (significant for GA) - $O_{i}^{k}, i=\overline{1, m}$.

- The method of estimating the properties of the obtained data as a result of the action mode decision;

- Restriction to the output variables and control actions that reflect the requirements caused by the goal-oriented decision.

- Subjective decision making model, which is a set of relations describing the dependence of control actions, parameters, and disturbances on the output variables.

Let us introduce measures for the above-described components. They will be used to estimate the goal-oriented condition.

1. Environment impact estimate. Assume, that the goaloriented agent is able to factorize characteristics of the environment $X^{k}=\left\{x_{i}^{k}, i=\overline{1, N}\right\}$. GA estimates the influence of each factor with the help of the linguistic variable called level of factor $\mu^{k}\left(x_{i}^{k}\right): x_{i}^{k} \rightarrow[0,1]$.

Suppose, that to formalizee the distinguished factors influence on the results $O_{i}^{k}, i=\overline{1, m}$ the goal-oriented agent uses an approximation in the form of the conditionaction rules which are as follows:

$$
\begin{aligned}
& \text { If } x_{1} \text { is } A_{r 1}^{k} \text { and } x_{2} \text { is } A_{r 2}^{k} \ldots \text { and } x_{N} \text { is } A_{r N}^{k} \text {, then } \\
& O_{i}^{k}=f_{i r}^{k}\left(x_{1}, x_{2}, \ldots, x_{N}\right), r=\overline{1, R}, i=\overline{1, m}
\end{aligned}
$$

where $R$ - the number of condition-action rules, $r$ - number of the current rule, $O_{i}^{k}=f_{i r}^{k}\left(x_{1}, x_{2}, \ldots, x_{N}\right)$ - is a clear function reflecting the $\mathrm{CA}$ conceptions of functional connection of input factors with possible data for the $r$-th rule (the r-th particular model);

$A_{r i}^{k}$ is a fuzzy variable defined on $X^{k}=\left\{x_{i}^{k}, i=\overline{1, N}\right\}$. 
2. Modes of action familiar (available) to GA. On condition that $C_{j}^{k}$ is a function of the ambient environment parameters, taken into consideration by GA, and of the functional and morphological properties of a system, a set of assumptions of their possible values gives rise to the scenarios of a possible state of the environment, system functionality and possibility of morphological changes. The implementation of scenarios, for example by using rule (1), allows to form an idea of the possible outcome $O_{i j}^{k}, j=\overline{1, m^{k}}$ and, hence, determines the nonequivalence of the mode of action decision. The nonequivalence can be described as the confidence level of the mode of action application. This estimate can be described as a linguistic variable $\psi \underset{i j l}{k}\left(O_{i j l}^{k}, \quad C_{j l}^{k}\right) \in[0,1], \quad l=\overline{1, m^{k}}$.

This measure is an individual characteristic of GA and can be changed due to training and gaining experience.

3. Possible results for a given decision environment. When an agent makes a decision in a goal-oriented condition in order to obtain a result, his mode of action decision is connected with the quantitative estimation of the selected solution properties, as shown in [5].

The choice of properties and measure parameters depends entirely on GA. Let us represent the possible outcomes for a given environment of GA decision in terms of $O_{i}^{k} \in\left\{O_{i j}^{k}, j=\overline{1, J}\right\}$ where $O_{i j}^{k}$ is set of possible outcomes in selecting the $j$-th mode of action; $i \in I$ is a set of results taken into account by the $k$-th GA.

4. Value of the results. This indicator follows from the qualitative assumption that the GA is able to compare the benefits gained in the course of receiving different types of results with the applied labour inputs. The value of the $j$-th type result can be estimated with the following the linguistic variable $\varphi_{i}^{k}\left(O_{i}^{k}\left(C_{j}\right)\right) \in[0,1]$.

5. Efficiency in the context of outcomes. It is a linguistic variable expressing the GA individual assessment of the decision consequences in terms of costs: financial, material, labor and labor intensity. Level of confidence $E_{i j}^{k}$, that a mode of action $C_{j}^{k}$ will lead to result $O_{i}^{k}$ in the environment $S$, if the GA chooses it:

$E_{i j}^{k}=E_{i j}^{k}\left(O_{i}^{k} \mid A\right.$ chooses $C_{j}^{k}$ в $\left.S\right) \in[0,1]$, permits to estimate the efficiency of the chosen by the GA mode of action.

6. The estimate of goal-oriented condition value. So, it is necessary to define the value of the goal-oriented condition by the $i$-th result for the $k$-th GA in accordance with the rule of Sugeno [5]:

$$
E \varphi_{i}^{k}=\frac{\sum_{j \in J} \varphi_{i j l}^{k}\left(O_{i j l}^{k}\left(C_{i l}^{k}\right)\right) \bullet \psi_{i j l}^{k}\left(O_{i j l}^{k}, S_{i j l}^{k}\right)}{\sum_{l=1}^{m} \varphi_{i j l}^{k}\left(O_{i j l}^{k}\left(C_{i l}^{k}\right)\right)} .
$$

By analogy, one can estimate the value of the goal-oriented condition by efficiency for the $k$-th GA for the i-th type of results:

$$
E E_{i}^{k}=\frac{\sum_{j \in J} E E_{i j}^{k}\left(O_{i}^{k}\left(C_{i}^{k}\right)\right) \bullet \psi_{i}^{k}\left(C_{j}^{k}\right)}{\sum_{j \in J} \psi_{i}^{k}\left(C_{j}^{k}\right)} .
$$

7. The GA estimate of the goal-oriented condition desirability. It is carried out by the $i$-th outcome and efficiency of its achievement in the decision situation. It is given by a linguistic variable

$\chi_{i 1}^{k}=\chi_{1}^{k}\left(E \varphi_{i}^{k}\right) \in[0,1], \quad \chi_{i 2}^{k}=\chi_{2}^{k}\left(E E_{i}^{k}\right) \in[0,1]$.The

basis of the personality and organization interaction is the psychological and economic contracts. They reflect the significant expectations of a person and the consistent expectations of an organization. Consequently, it becomes possible to determine the following restrictions: $\sum_{i} \chi_{i 1}^{k}\left(E \varphi_{i}^{k}\right) \geq \chi_{1}^{0}$ and $\sum_{i} \chi_{21}^{k}\left(E E_{i}^{k}\right) \geq \chi_{2}^{0}$ where $\chi_{1}^{0}$ and $\chi_{2}^{0}$ are the goal-oriented agent's expectations from the organization, which reflect the balance between costs and rewards for the results achieved.

Under the fixed remuneration function and in accordance with the hypothesis of rational behavior the GA makes the following decision:

$$
\begin{aligned}
& P_{i}(S)=\operatorname{Arg} \max \left(E \varphi_{i}\left(s_{i}, c_{i}\right)\right) \\
& s_{i} \in S_{i}, c_{i} \in C_{i}\left(I_{t}^{i}\right), I_{t}^{i} \subseteq M, \\
& \chi\left(E \varphi_{i}\right) \geq \chi 0
\end{aligned}
$$

where $\chi_{0}$ is the CA assessment of maximum gain, which he could obtain by performing the other job. It is obvious that if $\chi\left(E \varphi_{i}\right)<\chi 0$, one ought to expect that GA will choose $c_{i}=$ 0 .

All these mean that there is a certain GA, who is conditioned by the desire to get some sort of result. He has available several alternative ways to achieve the result with different level of efficiency, his confidence in getting the desired result being significant.

\section{MODEL OF FUZZY DESCRIPTION OF THE AGENT'S GOAL-ORIENTED BEHAVIOR}

The goal is accomplished through the problems, the problems, in turn, are materialized through the results $[4,5$, 7]. The results determine the desired outcome for a short 
period of time $t_{1} \leq t \leq t_{2}$ in a specific environment of the decision s:

$$
\begin{gathered}
I(t, s)=\underset{t_{1} \leq t \leq t_{2}}{\operatorname{argmax}}\left\{E V_{k}^{t}\left(O_{k}^{t}, t, s, c_{k}^{t}\right) \mid\right. \\
\left.\mid O_{k}^{t} \in O, j \in J, s \in S, c_{k}^{t} \in C, t_{1} \leq t \leq t_{2}\right\} .
\end{gathered}
$$

The problem is the last agent's desired outcome in the sequence of results: $I(t, s), t_{1} \leq t_{i} \leq t_{m}$, with $I_{1}\left(t_{1}, s_{1}\right)<I_{i}\left(t_{i}, s_{i}\right)<\ldots<I_{m}\left(t_{m}, s_{m}\right)$. That is the problem is described by the following formula: $\left.Z\left(t_{m}, s_{m}\right)=\max _{t_{1}<t<t_{m}} I_{k}(t, s) \mid t_{1} \leq t \leq t_{m}, s \in S\right\}$.

Goal is unattainable outcome $W$. But it can be infinitely closer in the problem solution sequence $Z_{v}\left(t_{v}, S_{v}\right)$ over a period of time $t_{1} \leq t \leq t v<t n$, with $Z_{1}\left(t_{1}, S_{1}\right)<$ $<Z_{2}\left(t_{2}, S_{2}\right)<\ldots<Z_{v}\left(t_{v}, S_{v}\right)<Z_{n}\left(t_{n}, S_{n}\right)=W^{*}$. Plausibility of achieving the goal $P_{v}\left\{Z\left(t_{v}, S_{v}\right) \geq Z_{n}\left(t_{n}, S_{n}\right)\right\}$ (the membership function of plausibility) comes arbitrarily close tol as $n$ increases. This exposition follows from the property of progressiveness of the goal-oriented intelligent system $[2,7]$.

In the course of making one's way to the problem the expected specific value, as regard to $O_{k}^{t}$, increases monotonically over the interval of time $t_{1} \leq t \leq t_{2}$ and so it does in the course of achieving the goal over the time interval

$$
t_{1} \leq t \leq t v<t_{m}
$$

$I_{m}\left(t_{m}, s_{m}\right)>I_{m-1}\left(t_{m-1}, s_{m-1}\right)$

and

$Z_{v}(t v, S v)>Z_{v-1}\left(t_{v-1}, S_{v-1}\right)$.

In the course of achieving the goal and the task a lot of available modes of agent actions and a set of possible outcomes can be recorded. When it is required in the course of advancing to construct novel modes of action in addition to old ones, their on-line search is applied. Subsequently

$$
\begin{aligned}
& I(t, s)=\underset{t_{1} \leq t \leq t_{2}}{\arg \max }\left\{E V_{j k}\left(O_{j}, t, s\right) \mid O_{j}\left(C_{i}\right) \in O,\right. \\
& \left.j \in J, O \in\{O\}, C_{i} \in C, i \in I, C \subset C^{\prime}, s \in S\right\}
\end{aligned}
$$

\section{Agent Conception Simulation Model of} GOAL-ORIENTED DECISION

The goal-oriented condition may include: $M$ - constraints, $C$ - action modes, $i$ - normative or ideal elements, $i e$ - the symbolic expression of normative or ideal elements, $\Omega$ - the external environment. In the agent's mind the condition exists in the form of conceptions and the agent forms them as the following components of the goal-oriented situation:

- accessible action modes;

- possible action mode outcomes;

- possible conditions of the decision environment (the possible values of uncontrollable variables which may affect accessible action mode outcomes including other agents' conceptions)

- probabilities that each possible condition of the decision environment turns out to be true;

- the efficiency of every accessible action mode in each possible outcome in every possible condition of the decision environment;

- $\quad$ specific value of every possible outcome.

The specific character of the agent conception simulation is the following. To modify his conceptions of some uncertain parameter the agent can use not only the results of monitoring the environment but also the results of monitoring the other agents' actions and action outcomes trying to "explain" their decision on the definite actions.

Let $\theta \in \Omega$ be a state of nature about which the agent with $k \in K$ number has interval information $\omega_{k}(\theta) \subseteq \Omega$, i.e. $\forall \theta \in \Omega, \forall k \in K, \quad \theta \in \omega_{k}(\theta)$.

Result of the system $O=G(\theta, c)$ depends on the vector $c=$ $\left(c_{1}, c_{2}, \ldots, c_{k}\right) \in C^{\prime}=\prod_{k \in K} C_{k}$ of other agents' actions connected with the $k$-th agent, where $c_{i} \in C_{i}$ and $\theta$ is a state of nature.

Assume that the specific value of each agent's decision depends on the state of nature $\theta$ and the result of the system $o$ in whole: $E V_{k}(o)=f_{k}(\theta, G(c, \theta)), k \in K$. Let assume the set of agents $K$, their outcomes $\left\{\mathrm{O}_{\mathrm{k}}(\bullet)\right\}$, sets of accessible action modes $\left\{\mathrm{C}_{\mathrm{k}}\right\}$, the set of possible nature state values $\Omega$, the function $G$ be common knowledge. Each agent observes the vector of all agents' actions, the overall result and all agent's results.

Since the overall result and specific value of each agent's outcomes depend on all agents' actions the apparatus of game theory can be used to describe their behavior.

Let the set of parametric Nash equilibriums (a parameter is the state of nature value) be

$$
\begin{gathered}
E_{N}(\theta)=\left\{\left\{c_{k}\right\} k \in K \in C^{\prime} \mid \forall k \in K, \forall x_{i} \in C_{k}\right. \\
f_{k}\left(\theta, G\left(\theta\left(c_{1}, \ldots, c_{k}\right)\right) \geq f_{k}\left(\theta, G\left(\theta, c_{1}, \ldots,\right.\right.\right. \\
\left.\left.\left.c_{i-1}, x_{i}, \ldots, c_{i+1}, \ldots, c_{k}\right)\right)\right\}
\end{gathered}
$$

If the set of $\Omega_{0} \subset \Omega$ of possible state of nature values is common knowledge for agents, then assuming their eliminating the uncertainty by computing the maximum 
guaranteed result we obtain the following set of their game equilibriums:

$$
\begin{aligned}
& \quad E\left(\Omega_{0}\right)=\left\{\left\{c_{k}\right\} k \in K \in C^{\prime} \mid \forall k \in K, \forall x_{k} \in C_{k}\right. \\
& \min _{\theta \in \Omega_{0}} f_{k}\left(\theta, G\left(\theta\left(c_{1}, \ldots, c_{k}\right)\right) \geq\right. \\
& \left.\geq \min _{\theta \in \Omega_{0}} f_{k}\left(\theta, G\left(\theta, c_{1}, \ldots, c_{i-1}, x_{i}, \ldots, c_{i+1}, \ldots, c_{k}\right)\right)\right\}
\end{aligned}
$$

Let $\mathrm{q}(\mathrm{c}) \subseteq \Omega$ be a set of nature states at which the action mode vector observed by agents is an equilibrium:

$$
q(c)=\left\{\theta \in \Omega \mid \exists \Omega_{0}: \theta \in \Omega_{0}\right\} .
$$

Let $g=\left(g_{1}, g_{2}, \ldots, g_{n}\right) \in \mathfrak{R}^{n}$ be the vector of their outcome values, with the outcomes being considered by them as satisfactory.

Let the set of nature state values, at which (along with the observed outcome $o$ ) the maximum specific values of the decision results of the agents $g$, be

$$
\eta(g, o)=\left\{\theta \in \Omega \mid f_{k}(\theta, z)=g_{k}, k \in K\right\} .
$$

Then, since the vector $g=\left(g_{1}, g_{2}, \ldots, g_{n}\right) \in \Re^{n}$ is determined by $E V_{k}$ for each $k \in K$, the vector $V=\left\{v_{1}, \ldots, v_{k}\right\} \in \Theta^{n}$ will determine the required level of agents' conceptions in a goal-oriented decision.

Based on [7] assume that the agent is subjectively rational: 1) his interests are expressed in subjective assessments of the value $\varphi_{i j}\left(O_{i}\left(C_{j}\right)\right) \in[0,1]$ of the expected outcomes $O_{i}, i=\overline{1, n}$ of the action modes $C_{j}(\theta, E), j=\overline{1, m}$ on the basis of the goal-oriented condition $\Omega ; 2$ ) the rationality of the agent's behavior consists in striving to maximize the specific value of his expectations. The decision on the agent's action mode is carried out under incomplete information and uncertainty.

Making an analysis the agent forms his own view of the decision situation in a hypothesis form attaching various measures of significance to the observed parameters and classifying them as for- or against- evidences. Besides the agent's efforts aim at both searching for the evidences proving the hypothesis proposed and searching for the evidences refuting it. Meanwhile, he does not use the whole information but only the one he considers to be necessary and sufficient for understanding the processes in the subject area.

The factors refuting the hypothesis make the agent either modify or revise it and include the positive aspects of the old one. The data analysis allows generating questions (queries) to confirm the assumptions, searching information for the answer being the basis for accepting or rejecting the original conceptions. This strategy allows forming consistent relationship between the parameters observed and the agent's conceptions under conditions of incomplete and unreliable information.

Definition 1. The agent is confident of his conception adequacy in the decision situation of $\Omega$ type relative to goal $\mathrm{G}$, if he believes that the action mode decision $C$ in accordance with his conceptions helps to achieve it. In this case: 1) he apprehends a part of characteristics $X$ of the decision situation $\Omega ; 2$ ) as for the other part he makes assumptions and shows the intention to prove (verify) their credibility; 3 ) in similar decision situations of $\Omega$ type in which he apprehended the presence (absence) of $\mathrm{X}$ and pursued $\mathrm{G}$, choosing $\mathrm{C}$ he always achieved $\mathrm{G}$; 4) observing the absence (presence) of $\mathrm{X}$ in a decision situation of $\Omega$ type, he would never choose $C$ to achieve the goal $G$ on basis of the conception.

The assumption is considered to be the default value of the characteristic observed or description of a cause-effect relation between the observable characteristics.

The agent's conceptions are characterized by the level of confidence.

Definition 2. The agent's level of confidence in his conceptions of the decision situation of $\Omega$ type relative to goal $G$ is determined by the frequency of its achievement when choosing the action mode $C$ on its base.

The level of confidence rating varies from zero to one. If the number of unsuccessful attempts to achieve the goal $G$ increases when the action mode $C$ is chosen according to the agent's conceptions, the agent's level of confidence decreases (and vice versa), which becomes an incentive for his making efforts to modify or reconstruct them completely as a result of increasing doubts about the credibility of the assumptions made.

The desire to verify the assumptions is a measure of the agent's doubts.

Definition 3. The efforts made by the agent to prove (disprove) the assumptions characterize the level of his doubts about the $\Omega$ decision situation conceptions when he pursues the goal $G$.

According to the behaviour psychology theory if the level of confidence, which, by definition 2, depends on the number of correct (according to conceptions) decision evidences, increases, the agent's desire to verify them decreases as he sees no sense in it. On the other hand, the agent's level of doubt increase is an incentive to search for additional arguments (counterarguments).

The parameter taking into account these two characteristics is the level of confidence being regarded as $S u=U_{u}^{\alpha} *\left(1-S_{S}\right)^{\beta} \in[0,1], \alpha+\beta=1$, where $S u$ is the degree of confidence; $U_{u}$ is the level of confidence (past experience); $S_{S}$ is the degree of the agent's doubt about his decision situation conception validity; $\alpha$ and $\beta$ are the coefficients of significance which the agent attaches to his experience and the need of evidence search. 
Assumption 1. Developing their views in this or that the agents use the argument apparatus to construct a sequence of hypotheses coming to the subjectively true one according to their conviction [6].

Assumption 2. Considering the occurred event $A$ the agent believes that it is caused by the event $B$. On the other hand, watching the event $A$ he makes a conclusion about the possibility of the event $\mathrm{B}$.

The $k$-th agent has the following sources of information about the environment [10]:

1) a priori private information $\omega_{k}(\theta) \subseteq \Omega$ (in the literature this type of information is referred to as knowledge, experience);

2) the other agents' actions: observing them and assuming that the opponents act rationally the agent may (under the common knowledge assumption) evaluate information $q(c)$ on the state of nature based on which the action mode decision was by the $i$-th agent $(i \neq k)$;

3 ) the set $g$ of the agent's decision results: - based on this information the agents can make a conclusion about the state of nature where the observed result leads to the observed gain;

4) the set $\rho \subseteq \Omega$ of the states of nature at which the vector of the agents' actions does result in the observed value $o$ of the system outcome:

$$
\sigma(c, o)=\{\theta \in \Omega \mid G(\theta(c)=o\} .
$$

According to the assumption made above the information 2 -4 is the agents' common knowledge. Then the relation $I(c$, $o, g)=q(c) \cap \sigma(x, o) \cap \eta(g, o) \subseteq \Omega$ is true. Based on common knowledge and his own conceptions each $k$-th agent can estimate $J_{k} \in \Omega$ of the state of nature value as the intersection of the common knowledge $I(c, o, g)$ and his private information $\omega_{k}$ :

$$
J_{k}\left(\omega_{k}, c, o, g\right)=\omega_{k} \cap I(c, o, g)[10] .
$$

Let $c_{k}^{t} \in C_{k}$ be an action mode of the $k$-th agent to get a result at step $t, c t$ be a set of all the agents' action vectors in $t$ steps. At the moment of getting a result at step $t$ the agents' common knowledge will be

$$
I\left(c^{t}, o^{t}, g t\right)=q\left(c^{t}\right) \cap \sigma\left(c^{t}, o^{t}\right) \cap \eta\left(g^{t}, o^{t}\right) \subseteq \Omega .
$$

Based on all the information sources the $k$-th agent within $t$ periods can estimate the state of nature value $J_{k}^{t} \subseteq \Omega$ as the intersection of the common knowledge $I\left(c^{t}, o^{t}, g^{t}\right)$ and his private information $J_{k}^{t-1}$ corresponding to the previous period:

$$
J_{k}^{t}=J_{k}^{t-1} \cap I\left(c^{t}, o^{t}, g^{t}\right)
$$

Owing to the specific value increase when the agent moves to the goal achievement and in accordance with the properties of the agent's conception generation $v_{k}^{t}\left(J_{k}^{t}\right)<v_{k}^{t-1}\left(J_{k}^{t-1}\right)$, where $v_{k}^{t}$ are the estimates of the agent's confidence in his conception structure adequacy.

Thus, under the hypothesis of rational behavior and the hypothesis of common knowledge the agent's convergent conception generation will be observed.

\section{INFORMATION AWARENESS EVALUATION IN FUZZY CHOICE PROBLEM}

In the situation of incomplete information, knowledge or lack of time the agent builds a model of the subject area temporarily proceeding from the plausible assumptions about which he does not have sufficient evidence (e.g., availability of the supporting information) $[8,9]$. The set of such assumptions, ideas and views aims at explaining the phenomena, processes and relationship between them in a particular subject area and they form a hypothetical concept of an agent which is thought to be hypothetical, subjective knowledge.

The conclusions made on hypothetical concepts (a subjective theory) identify a decision-maker has a condition of confidence in the subjective understanding of a goaloriented condition. The condition stability depends on the frequency and duration of successful outcomes relative to the goal $(O)$ at the expense of the adopted precondition and hypotheses truth, the validity of the conclusion generation rules (i.e., a hypothetical concept in general).

If the result obtained from making the decision formed according to the decision-maker's subjective conceptions does not meet his expectations and generates a condition of doubt about the goal-oriented condition, he revises his views in the non-monotonic way, which involves removing a mistaken precondition and / or introducing a new one, as well as the inference rule correction. Thus, new views are the outcomes of new information obtained from the conclusion system and decision monitoring system.

Definition 4. Let fuzzy sets be $A \subseteq X$ and $B \subseteq X$, where $\mathrm{X}$ is a crisp set. Assume that for each fuzzy set $\alpha$-level are defined sets in the following way $A \alpha=\left\{x \in X: \mu_{A}(x) \geq \alpha\right\}, B \alpha=\left\{x \in X: \mu_{B}(x) \geq \alpha\right\}$, where $\mu_{A}(x)$ and $\mu_{B}(x)$ are membership functions which values express the agent's level of confidence in membership of the element $\mathrm{x}$ in $A$ and $B$ sets respectively. Then the alternative $a$ would be preferable to the alternative $b$ if and only if $x_{a}>x_{b}, \forall x_{a} \in A \alpha(x), x_{b} \in B \alpha(x)$, i.e. $A$ is greater than $\mathrm{B}$ at $\alpha$ level.

Let $\alpha$ be the minimum value of $\alpha$ when the inequality $x_{\alpha}>x_{b}, \forall x a \in A_{\alpha}(x), x_{b} \in B_{\alpha}(x)$ is true. Then $1-\underline{\alpha}$ is the level of confidence in the preference for $a$ relative to $b$ and indifference in the choice of $a$ or $b$. By analogy, if $A_{\alpha}$ 
is contained in $B_{\alpha}$, i.e. $A \alpha \subseteq B \alpha$, we say that $A$ is contained in $B$ at level $\alpha$.

If the value of $\rho=1-\underline{\alpha}$ increases (or $\underline{\alpha}$ decreases), the statement that $A$ is greater than $B$ is true. If $\alpha=0$, any element belonging to a fuzzy set will, for sure, only belong to this set.

The introduction of level of confidence measure when the alternatives are compared allows:

- determining the level of information sufficiency for decision making. If the level of confidence value is below a certain threshold, the decision making is delayed to gather more information.

- determining for GA the additional information value. It can be zero if the level of confidence does not change after it is obtained. If the value $\rho=1-\alpha$ increases, the information contributes to the formation of the GA's views on the decision situation. If $\rho_{i}\left(\alpha_{i}\right)<\rho_{i-1}\left(\alpha_{i-1}\right)$, either there is misinformation, or the data obtained destroy the GA's views on the decision situation and new data are required.

The value $\Delta \rho_{i}=\rho_{i}-\rho_{i-1}>0(<0)$ allows determining the direction of the information search. Suppose there are two statements: $\rho \cong X$ is $G$ and $\mathrm{q} \cong X$ is $F$, where $F$ and $G$ are predicates represented as fuzzy sets. Then, $G \subset F$, (p $\Rightarrow \mathrm{q}$ (p implies q). This means that the first statement is more informative than the second one.

Therefore, changing the GA's awareness leads to changing his conceptions and as a result to changing $\mu_{A}(x)$ and supp

$A$. They can be used as a measure of GA's awareness.

$\mathrm{G} \subset \mathrm{F}$ requires the increase in the number of properties taken into account in description. In addition, each property added should increase the level of confidence in distinguishing objects.

Increasing the number of properties can result in the two situations:

1. The new information increases the level of confidence in $\mathrm{G} \subset F$, i.e. the statement with a new property is more informative than the same statement without it.

2. If the two objects with the same number of evaluated properties are compared, and one more property is added, but its value of both objects is difficult to distinguish, then the extra information does neither increase the level of confidence in distinguishing the objects nor reduce it.

\section{CONCLUSIONS}

1. The model of rational choice has been extended by introducing subjective estimates of the decision situation components.

2. The linguistic measures of the decision situation property estimate have been proposed.

3. The convergent process of the agent's conception of the decision situation has been proved to exist with the rational choice.

4. The condition of subjective confidence of the agent's conception adequacy in the decision situation has been defined.

5. The procedure of nonmonotonic reasoning in generating the decision situation conceptions has been proposed.

\section{REFERENCES}

[1]. Novikov, D. A. Modern Problems of the Theory of Managing Organizational Systems / Human Factor in Management // Edited by N.A. Abramova, K.S. Ginsberg, D.A. Novikov. - M.: KomKniga, 2006. - P. 391-407. Akoff, R. On Goal-Oriented Systems / R. Akoff, F. Emery// Edited by A.I. Ushakov. Moscow: Sov. Radio, 1974. - 272p.

[2]. Vinogradov, G. P. Behavior Model of an Intelligent Agent // Integrated Models and Soft Computing in Artificial Intelligence // Collection of Scientific Papers of the V-th International Scientific Conference (Kolomna, May 28-30, 2009). - Moscow: Fizmatlit, 2009, pp. 443-455.

[3]. Vinogradov, G. P. Modeling of Decision-Making by Intelligent Agent / Software Products and Systems, 2010, № 3, pp. 45 - 51.

[4]. Vinogradov, G. P. Estimates of Conviction about the Level of Awareness of an Intelligent Agent in the Fuzzy Choice Problem / G.P. Vinogradov, N.V. Kirsanov, R.S. Fedotov // Collection of Scientific Papers of the Twelfth National Conference on Artificial Intelligence (with international participation) KII-2010 (20-24 September 2010, Tver, Russia). - Moscow: Fizmatlit, 2010, vol. 1. pp. $21-28$.

[5]. Burkov, V.N. Intelligent Active Systems / G.P. Vinogradov, V.N. Burkov, V.N. Kuznetsov, B.V. Palyukh, N.A. Semenov // Collection of Scientific Papers of the Twelfth National Conference on Artificial Intelligence (with international participation) KII-2010 (20-24 September 2010, Tver, Russia). Moscow: Fizmatlit, 2010, vol3, pp. 35 - 43.

[6]. Vinogradov, G.P. Simulation of the Intelligent Agent Conception of the Goal-Oriented Decision Condition / Problems of Computer Science, 2010, № 3, pp. 66-72.

[7]. Vinogradov, G.P. Model of Agent's Conception and Conviction on the Goal-Oriented Decision Condition // Proceedings of Congress on Intelligent Systems and Information Technology AIS-IT-10 / Scientific publication in 4 vols. - Moscow: Fizmatlit, 2010, volume 1, pp. 119-123.

[8]. Vinogradov, G.P. Matching Model of Agents' Conception to Intelligent System. // Izvestiya of JuFU. Technical Sciences, 2009, № 12 (101), pp. 109-114.

[9]. Novikov, D.A. Mathematical Models of Generating and Functioning of Commands. - Moscow: Fizmatlit, 2008,184p. 